\title{
Elucidation of Linker Motion in Metal-Organic Frameworks by Three-Dimensional Electron Diffraction
}

\author{
L. Samperisi ${ }^{1}$, A. Jaworski ${ }^{1}$, G. Kaur ${ }^{2}$, K. P. Lillerud ${ }^{2}$, X. Zou ${ }^{1}$, Zhehao Huang ${ }^{1}$ \\ ${ }^{1}$ Department of Materials and Environmental Chemistry, Stockholm University, Stockholm SE-106 91, Sweden \\ ${ }^{2}$ Department of Chemistry, University of Oslo, P.O. Box 1033, N-0315 Oslo, Norway \\ laura.samperisi@mmk.su.se
}

The sensitiveness to the electron beam of nanocrystalline metal-organic frameworks (MOFs) has always posed an objective criticality for the accurate determination of their structure by single crystal electron diffraction. The reversible atomic displacement caused by the high flexibility of the organic linkers further complicates the characterization of the framework and the understanding of their complex functional properties at the atomic level ${ }^{[1]}$. Although standard diffraction experiments can elucidate dynamic phenomena ${ }^{[2,3]}$, an analysis of the anisotropic displacement parameters (ADPs) obtained after refining MOFs against electron diffraction data has never been performed. In this study, we solved and refined the structures of UiO-67/MIL-140C, coexisting in mixture, by using continuous rotation electron diffraction (cRED). For both structures, restricted small-angle librations of the linker were revealed by analysing the ADPs at room temperature and in cryogenic conditions $(98 \mathrm{~K})$. Our work shows that continuous rotation electron diffraction (cRED) not only provides reliable and accurate crystallographic models as that obtained by single crystal X-ray diffraction (SCXRD), but it represents a powerful tool to investigate the dynamic in the molecular fragments of the framework.

[1] Bennett, T.; Cheetham, A.; Fuchs, A.; Coudert, F.-X. (2017) Nature Chem., 9, 11-16

[2] Smeets, S.; Parois, P.; Burgi, H- B; Lutz, M. (2011) Acta Cryst, B67, 53-62

[3] Lock, N.; Wu, Y.; Christensen, M.; Cameron, L. J.; Peterson, V. K.; Bridgeman, A. J.; Kepert, C. J.; Iversen, B. B. (2010) J. Phys. Chem. C, 114, $16181-16186$

Keywords: ADPs, flexibility, MOFs, cRED 